\title{
EXISTEM DIFERENÇAS ENTRE OS DIURÉTICOS TIAZÍDICOS?
}

\author{
ARE THERE ANY DIFFERENCES BETWEEN THIAZIDE DIURETICS?
}

\author{
Fernando Focaccia Póvoa', Rui Póvoa ${ }^{2,3}$
}

\section{RESUMO}

Desenvolvidos em 1950, os diuréticos estão entre as drogas mais utilizadas no arsenal terapêutico clínico, principalmente na hipertensão arterial e nos quadros edematosos. O mecanismo de ação envolve a excreção renal de água, eletrólitos e diminuição da reabsorção de sódio em diferentes locais do néfron, com consequente aumento do sódio urinário e da água. Os diuréticos tiazídicos mais usados na prática clínica em pacientes hipertensos são: hidroclorotiazida (HCTZ), clortalidona (CTD) e indapamida (IDP). Em relação à potência anti-hipertensiva, a CTD é uma vez e meia a duas vezes mais potente que a hidroclorotiazida. HCTZ é menos potente do que qualquer outro anti-hipertensivo, incluindo inibidores da enzima de conversão da angiotensina, bloqueadores do receptor da angiotensina e antagonistas dos canais de cálcio. O IDP tem ação anti-hipertensiva devido aos seus efeitos no túbulo distal, inibindo a reabsorção do cloreto de sódio e tem efeitos mais intensos e sustentados na redução da pressão arterial. Os diuréticos tiazídicos são muito diferentes, tanto em níveis variados de redução da pressão arterial quanto em efeitos adversos, de modo que sua proteção contra danos em órgãos-alvo está relacionada ao efeito de classe.

Descritores: Diuréticos; Clortalidona; Hidroclorotiazida; Indapamida.

\section{ABSTRACT}

Developed in 1950, diuretics are among the most used drugs in the clinician's therapeutic arsenal, especially in arterial hypertension and edematous conditions. The mechanism of action involves the renal excretion of water, electrolytes, and decreasing the reabsorption of sodium in different locations of the nephron, with consequently an increase in urinary sodium and water. The most used thiazide diuretics in clinical practice in hypertensive patients are: hydrochlorothiazide (HCTZ), chlortalidone (CTD) and indapamide (IDP). Regarding the antihypertensive potency, CTD is one and a half to two times more potent than hydrochlorothiazide. HCTZ is less potent than any other antihypertensives including angiotensin converting enzyme inhibitors, angiotensin receptor blockers, and calcium channel antagonists. IDP has an antihypertensive action due to its effects on the distal contoured tubule, inhibiting sodium chloride reabsorption and has more intense and sustained effects in reducing blood pressure. Thiazide diuretics are very different, both in varying levels of blood pressure reduction and adverse effects, so their protection from target organ damage is related to the class effect.

Keywords: Diuretics; Chlortalidone, Hydrochlorothiazide, Indapamide.

Os diuréticos são os fármacos mais utilizados na cardiologia, em vista da importância terapêutica reduzindo sintomas e diminuindo sensivelmente a mortalidade cardiovascular. Seu uso isolado ou em combinação com outros fármacos, os diuréticos, foram os pioneiros no tratamento de diversas patologias cardiovasculares, destacando a hipertensão arterial e a insuficiência cardíaca com importante expressividade na prática clínica. Destaque em diversos estudos com redução da morbimortalidade cardiovascular quando utilizado isoladamente ou em combinação com outros fármacos de ação cardiovascular. Desenvolvidos em 1950, estão entre as drogas mais utilizadas no arsenal terapêutico do clínico, principalmente na hipertensão arterial e nos quadros de edematosos. ${ }^{1,2}$
O mecanismo de ação envolve a excreção renal de água, eletrólitos, diminuindo, a reabsorção de sódio $\left(\mathrm{Na}^{+}\right)$em diferentes locais do néfron, com aumento, consequentemente, do sódio urinário e de água. ${ }^{3}$

Podemos dividi-los de acordo com seu local de ação em: 1. diuréticos de alça que atuam diminuindo a reabsorção do $\mathrm{Na}^{+}$no ramo espesso da alça de Henle, por inibir o sistema de cotransporte $\mathrm{Na}^{+}-\mathrm{K}^{+}-2 \mathrm{Cl}$ - que se localiza na membrana apical do túbulo renal; 2. tiazídicos e correlatos que atuam inibindo a reabsorção de sódio na porção inicial do túbulo contornado distal; 3. poupadores de potássio que inibem a reabsorção de $\mathrm{Na}^{+}$, reduzindo seu intercâmbio com o $\mathrm{K}^{+}$e deste modo diminuindo a eliminação de $\mathrm{K}^{+}$e os4.osmóticos e inibidores da anidrase carbônica. ${ }^{1-3}$ 
Os diuréticos tiazídicos mais utilizados na prática clínica nos pacientes hipertensos são hidroclorotiazida (HCTZ), a clortalidona (CTD) e a indapamida (IDP). Apesar da similaridade dos mecanismos de ação há uma grande discussão se eles são semelhantes entre si ou diferem em algo.

Em relação a potência anti-hipertensiva dados sugerem que a clortalidona é de uma vez e meia à duas vezes mais potente do que a hidroclorotiazida. ${ }^{4} \mathrm{~A}$ HCTZ deverá ser usada na dose de $37,5 \mathrm{mg} /$ dia para alcançar a mesma potência hipotensora que a CTD na dose de $12,5 \mathrm{mg}$ a $25,0 \mathrm{mg} / \mathrm{dia} .^{5}$

Vongpatanasin $W$ et al. em 2015, realizaram uma revisão sistemática para determinar a utilidade e versatilidade da HCTZ em relação aos demais diuréticos tiazidicos no tratamento da hipertensão arterial. ${ }^{6} \mathrm{~A}$ HCTZ foi considerada menos potente na redução da pressão arterial do que os outros diuréticos tiazídicos, incluindo a CTD e a bendroflumetiazida.

A análise também verificou que HCTZ (12,5-25 mg por dia) é menos potente do que quaisquer outros anti-hipertensivos incluindo inibidores da enzima de conversão da angiotensina, bloqueadores do receptor da angiotensina e antagonistas dos canais de cálcio. O risco de hiponatremia, hipocalemia, e hiperuricemia associada com HCTZ foi menor do que com a CTD enquanto o risco de artrite gotosa foi semelhante. Apesar dos riscos mais baixos de efeitos colaterais metabólicos esta revisão sistemática encontrou que para qualquer redução na PA sistólica alcançada, a terapia com HCTZ foi associada com $18 \%$ a mais de eventos cardiovasculares em relação a CTD. ${ }^{6}$

Na Tabela 1 observamos as principais diferenças entre a HCTZ e a CTD.

A indapamida é um diurético "tiazida-like", pois não possui o anel tiazídico em sua composição molecular. Possui ação anti-hipertensiva por efeitos no túbulo contornado distal inibindo a reabsorção de cloreto de sódio, e também ações diretas na inibição da hiper-reatividade das aminas vasoativas. Também desencadeia aumento das prostaglandinas vasodilatadoras, inibição da síntese de tromboxane $A_{2}$ e inibição do fluxo de íons cálcio nas fibras musculares lisas, reduzindo a resistência vascular periférica e os níveis pressóricos. ${ }^{7}$

Apesar da semelhança com os outros diuréticos do grupo tiazida, a indapamida tem características únicas em termos de eficácia e ação anti-hipertensiva, com efeitos mais intensos e sustentados em reduzir a pressão arterial. Em comparação com os outros tiazídicos, além de menor

Tabela 1. Comparação da eficácia e segurança da Hidroclorotiazida (HCTZ) versus clortalidona (CTD).

\begin{tabular}{l|c|c}
\multicolumn{1}{c|}{ Efeitos } & HCTZ & CTD \\
Meia vida & $5-15 \mathrm{~h}$ & $30-72 \mathrm{~h}$ \\
\hline Hipocalemia & $\uparrow$ & $\uparrow \uparrow$ \\
\hline Hiponatremia & $\uparrow$ & $\uparrow \uparrow$ \\
\hline Hiperuricemia & $\uparrow$ & $\uparrow \uparrow$ \\
\hline Redução da PA consultório & $\downarrow$ & $\downarrow \downarrow$ \\
\hline Redução da PA noturna & $\downarrow$ & $\downarrow \downarrow$ \\
\hline $\begin{array}{l}\text { Efeitos pleiotrópicos (antiplaquetários } \\
\text { e vasculares) }\end{array}$ & não & $\operatorname{sim}$ \\
\hline Proteção Cardiovascular & $\uparrow$ & $\uparrow \uparrow$
\end{tabular}

PA: pressão arterial, $\uparrow=$ aumento leve, $\uparrow \uparrow=$ aumento moderado $\downarrow=$ redução leve, $\downarrow \downarrow=$ redução moderada. Adaptado da referência 6. incidência de hipocalemia e hiperglicemia, é praticamente neutro em provocar distúrbios metabólicos. ${ }^{8}$

Ambrosioni et al. avaliando a indapamida na dose de 1,5 e 2,5 mg em 650 pacientes hipertensos, verificaram a eficácia anti-hipertensiva destas baixas doses, sem ação significante no metabolismo. ${ }^{9}$

Leonetti el al. avaliaram a eficácia e a tolerabilidade da indapamida em hipertensos idosos. Em um acompanhamento de 12 meses de 444 pacientes em uso da indapamida, verificou-se uma redução de $24,0 \mathrm{mmHg}$ para a sistólica e de $13,1 \mathrm{mmHg}$ para a diastólica. A porcentagem dos pacientes que atingiram a meta foi de $80,1 \%$, com boa tolerabilidade. A taxa de abandono foi somente de $7,2 \% .^{10}$

Para o lado de proteção renal, Madkour et al. compararam a indapamida em hipertensos com lesão renal já estabelecida, e verificaram que de forma similar a HCTZ reduziam igualmente a pressão arterial. Entretanto a indapamida foi superior em melhorar o clearence de creatinina, com diferenças estaticamente significantes. O grupo que utilizou a indapamida teve melhora de $28,5 \pm 4,4 \%$ no clearence versus $17,4 \pm 3,0 \%$, $(p<0,05)$, do grupo HCTZ. ${ }^{11}$

A indapamida é eficiente em reduzir a microalbuminúria em idosos hipertensos com diabetes. No estudo NESTOR (Natrilix SR versus Enalapril Study in hypertensive Type 2 diabetics with micrOalbuminuRia) foi comparado a indapamida com o enalapril em 570 pacientes e houve redução da taxa urinária de albumina/creatinina de $46 \%$ com a indapamida e de $47 \%$ com o enalapril, mostrando assim a não inferioridade deste diurético com um fármaco inibidor do sistema renina angiotensina aldosterona. ${ }^{12}$

Mogensen et al., ${ }^{12}$ compararam a associação da indapamida $(0,625 ; 1,25$ e $2,5 \mathrm{mg})$ associada ao perindopril (2,4 e 8,0 mg) versus enalapril (10, 20 e $40 \mathrm{mg})$, nos efeitos sobre a taxa de excreção da albumina em diabéticos e encontraram importante decréscimo na excreção de albumina, na associação indapamida e perindopril. A redução pressórica foi semelhante nos dois grupos, entretanto na associação a taxa de excreção de albumina caiu $42 \%$ versus $27 \%$ do grupo enalapril $(p=0,01) \cdot{ }^{13}$

No estudo ADVANCE a indapamida associada com o perindopril foram avaliados na redução de eventos macro e microvasculares (morte, infarto do miocárdio não fatal, piora da função renal, desenvolvimento da macroalbuminúria, e morte de causa renal) em 11.140 diabéticos em um período médio de 4,3 anos. O estudo mostrou que a indapamida mais o perindopril reduziu significantemente eventos cardiovasculares, a microalbuminúria, todas as causas de mortalidade e menos mortes cardiovasculares. ${ }^{14}$

A hipertrofia ventricular esquerda, principal lesão cardíaca decorrente da hipertensão, é um outro fator de risco independente de morbimortalidade cardiovascular. Consequentemente todo anti-hipertensivo que se preze necessita reduzir a massa ventricular.

Tanto da HCTZ quanto a CTD são importantes em termos de redução da massa ventricular esquerda, por mecanismos ainda não muito bem esclarecidos, porém a redução pressórica participa de forma fundamental neste processo. Isto foi muito bem avaliado em diversas metanálises comparando os diuréticos com os outros fármacos anti-hipertensivos. 
Entretanto a comparação entre os tiazídicos na redução da massa cardíaca ainda carece de estudos robustos. ${ }^{15}$

A indapamida também está associada com importante redução da massa ventricular esquerda. Gosse et al. comparando a eficácia da indapamida 1,5 mg versus o enalapril na redução da massa ventricular esquerda, verificaram que era muito mais efetiva na redução da massa ventricular $(8,4 \pm 30,5$ g versus $1,9 \pm 28,3 g, p=0,013)$, apesar da redução dos níveis pressóricos serem semelhantes. Estes efeitos da indapamida foram progressivos e se mantiveram após um ano, fato que não ocorreu com o enalapril. ${ }^{16}$

No estudo PROGRESS os 6.105 pacientes com história de acidente vascular cerebral (AVC) e ataque isquêmico transitório, foram randomizados para a utilização do perindopril com ou sem indapamida. O grupo em que estava associado ao diurético apresentou redução expressiva da pressão arterial, com redução do risco de AVC de 43\%. A combinação do IECA com a indapamida, além da ação anti-hipertensiva, reduziu de forma significante os episódios de AVC. ${ }^{17}$

Na prevenção de insuficiência cardíaca o estudo ALLHAT mostrou que a CTD quando comparada com a amlodipina e lisinopril foi a mais efetiva em reduzir a insuficiência cardíaca. ${ }^{18}$
De todos os ensaios clínicos envolvendo a indapamida com certeza o estudo HYVET foi o mais importante, em vista de envolver o tratamento da hipertensão arterial em muito idosos. Foram randomizados pacientes hipertensos hígidos com mais de 80 anos e a utilização da indapamida titulada com perindopril mostrou redução da AVC de $30 \%$ sendo os objetivos primários, apesar de não ter significância estatística $(p=0,06)$. Entretanto o estudo foi interrompido precocemente devido aos resultados dos objetivos secundários, onde a combinação medicamentosa reduziu todas as causas de mortalidade em $21 \%(p=0,02)$, AVC fatal em 39\% $(p=0,04)$ e principalmente a redução expressiva da insuficiência cardíaca reduzindo em $64 \%(p<0,001) .{ }^{19}$

Concluindo e respondendo à pergunta, o diauréticos tiazidas são muito diferentes, tanto em níveis variados de redução da pressão arterial quanto aos efeitos adversos. Os distúrbios para o lado glicêmico e lipídico, além da esfera dos íons são expressivos para a HCTZ e CTD, ficando a indapamida neutra. Também existe variações em relação a proteção as lesões em órgãos alvo, documentada em diversos estudos, porém o aspecto protetor está mais relacionada a classe e se reflete primordialmente na ação anti-hipertensiva.

\section{REFERÊNCIAS}

1. Nigro D, Fortes ZB. Efeitos farmacológicos dos diuréticos e dos bloqueadores dos canais de cálcio. Rev Bras Hipert. 2005,12(2):103-5.

2. Florez J, Armijo J A, Mediavilla A. Fármacos diuréticos. In: Florez J, Armijo J A, Mediavilla A (eds). Farmacologia humana. Eunsa, Pamplona, 632-639, 1988.

3. UpTo Date. Brater DC. Mechanism of action of diuretics. Last literature review version 19.3. This topic last updated November 15, 2011. www. uptodate.com/contents/mechanism-of-action-of-diuretics

4. Ernst ME, Carter BL, Goerdt CJ, et al. Comparative Antihypertensive Effects of Hydrochlorthiazide and Chlorthalidone on Ambulatory and Office Blood Pressure Hypertension. Hypertension. 2006;47:352-8.

5. Grossman E, Verdecchia P, Shamiss A, Angeli F, Reboldi G. Diuretic Treatment of Hypertension. Diabetic Care. 2011;34( suppl2):s313-s319.

6. Vongpatanasin W. Hydrochlorothiazide (HCTZ) is not the most useful nor versatile thiazide diuretic.Curr Opin Cardiol. 2015; 30(4): 361-5.

7. Sassard J, Bataillard A, Mclntyre H. An overview of the pharmacology and clinical efficacy of indapamide sustained release. Fundam Clin Pharmacol 2005; 19:637-45.

8. Al Badarin FJ, Abuannadi MA, Lavie CJ, et al. Evidence-based diuretic therapy for improving cardiovascular prognosis in systemic hypertension. Am J Cardiol. 2011;107:1178-84.

9. Ambrosioni E, Safar M, Degaute JP, et al. Low-dose antihypertensive therapy with $1.5 \mathrm{mg}$ sustained-release indapamide: results of randomised doubleblind controlled studies. European study group. J Hypertens. 1998; 16: 1677-84.

10. Leonetti G, Emeriau JP, Knauf H, Pujadas JO, Calvo-Gomez C, Abate G; European Study Investigators. Evaluation of long-term efficacy and acceptability of indapamide SR in elderly hypertensive patients. Curr Med Res Opin. 2005; 21(1):37-46.
11. Madkour H, Gadallah M, Riveline B, Plante GE, Massry SG. Indapamide is superior to thiazide in the preservation of renal function in patients with renal insufficiency and systemic hypertension. Am J Cardiol. 1996;77(suppl):23B-25B.

12. Puig JG, Marre M, Kokot F, et al. Efficacy of indapamide SR compared with enalapril in elderly hypertensive patients with type 2 diabetes. Am J Hypertens. 2007; 20(1):90-7.

13. Mogensen CE, Viberti G, Halimi S, et al. Effect of low-dose perindopril/ indapamide on albuminuria in diabetes: Preterax in albuminuria regression: PREMIER. Hypertension. 2003;41:1063-71.

14. Patel A, MacMahon S, Chalmers J, et al. Effects of a fixed combination of perindopril and indapamide on macrovascular and microvascular outcomes in patients with type 2 diabetes mellitus (the ADVANCE trial): a randomized controlled trial. Lancet. 2007; 370:829-40.

15. Neaton J D Grimm Jr R H, Prineas R J, et al. Treatment of Mild Hypertension Study. Final results. Treatment of Mild Hypertension Study Research Group. JAMA. 1993;270(6):713-24.

16. Gosse P, Sheridan DJ, Zannad F, et al. Regression of left ventricular hypertrophy in hypertensive patients treated with indapamide SR $1.5 \mathrm{mg}$ versus enalapril $20 \mathrm{mg}$ : the LIVE study. J Hypertens. 2000;18:1465-75.

17. PROGRESS Collaborative Group. Randomised trial of a perindopril-based blood-pressure-lowering regimen among 6,105 individuals with previous stroke or transient ischaemic attack. Lancet. 2001;358:1033-41.

18. Oparil S, Davis BR, Cushman WC, et al. Mortality and Morbidity During and After Antihypertensive and Lipid-Lowering Treatment to Prevent Heart Attack Trial. Hypertension. 2013; 61:977-86.

19. Beckett NS, Peters R, Fletcher AE, et al. Treatment of hypertension in patients 80 years of age or older. N Engl J Med. 2008;358:1887-98. 